\title{
Combined Shearography and Speckle Pattern Photography for Single-Access Multi-Component Surface Strain Measurement
}

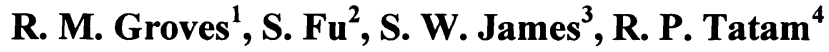 \\ 1. Optical Sensors Group, Centre for Photonics and Optical Engineering, \\ r.groves@cranfield.ac.uk. \\ 2. Centre for Computer Vision and Image Processing, s.fu@cranfield.ac.uk. \\ 3. Optical Sensors Group, Centre for Photonics and Optical Engineering, \\ s.w.james@cranfield.ac.uk. \\ 4. Optical Sensors Group, Centre for Photonics and Optical Engineering, \\ r.p.tatam@cranfield.ac.uk.
}

School of Engineering, Cranfield University, Cranfield, Bedfordshire, MK43 0AL, UK.

Corresponding Author: S. W. James

\begin{abstract}
Full surface strain measurement requires the determination of two out-of-plane and four in-plane displacement gradient components of the surface strain tensor. Shearography is a full-field speckle interferometry technique with a sensitivity predominately to the out-of-plane displacement gradient. Speckle pattern photography has the sensitivity to the in-plane displacement, and taking the derivative yields the in-plane displacement gradient. In this paper the two techniques are combined to yield a single-access multi-component surface strain measurement using shearography to measure the out-of-plane components and speckle pattern photography to measure the in-plane components. Results are presented of a multi-component surface strain measurement.
\end{abstract}

Keywords: Shearography, Speckle Pattern Photography, Surface Strain Measurement

\section{Introduction}

Speckle shearing interferometry, often termed shearography [1,2], is a full-field non-contact optical technique, usually used for the qualitative measurement of changes in the displacement gradient caused by surface and subsurface defects. A speckling pattern formed by illuminating an optically rough surface with an expanded coherent laser beam. In shearography this speckle pattern is optically mixed with an identical but displaced, or sheared, speckle pattern using a shearing device, and viewed through a lens, forming a speckle interferogram at the camera surface. The shearing device commonly used for shearography is a Michelson interferometer with a tilt applied to one of the mirrors [1]. Correlating speckle interferograms obtained before and after object deformation yields correlation fringes sensitive to displacement gradient in the shear direction. Phase-stepping techniques [3] are often used to extract the phase information, and therefore the displacement gradient information, from the speckle interferograms.

Speckle Pattern Photography [4] is a general term for image processing techniques that determine displacement of a speckle pattern in order to determine displacement and deformation fields. This paper uses the Optical Flow Field technique [5] to determine the displacement. In this technique it is assumed that intensity changes at individual camera pixels, before and after deformation, is due to the motion of the speckle pattern and that this motion of the speckle pattern is a direct result of displacement at the object surface. The advantages of this approach over the more conventional discrete correlation technique [4] are discussed. 
To fully characterise the surface strain requires the measurement of six components of displacement gradient. This is composed of two out-of-plane and four in-plane displacement gradient components. The shearography technique can directly measure the out-of-plane displacement gradient using illumination and viewing directions, which are collinear and normal to the object surface. The measurement of the in-plane displacement gradient components, using shearography, requires illumination, or viewing, of the object from a minimum of three directions and a coordinate transformation of the measured displacement gradient components [6]. Speckle pattern photography is sensitive to the in-plane displacement [7] and this parameter may be differentiated to yield the in-plane displacement gradient.

In this paper a combined shearography and speckle pattern photography instrument is described which can fully characterise the surface strain from a single viewing direction. Shearography is used to measure the out-of-plane components of displacement gradient and speckle pattern photography is used to measure the in-plane components of displacement gradient. Results are presented of surface strain measurement made with this combined instrument.

\section{Theory - Shearography}

Shearography is a full field optical technique that can be used for the measurement of displacement gradient. The component of displacement gradient that is measured by shearography is determined by the sensitivity vector, which is the bisector of the illumination direction and the viewing direction, and by the direction of the applied shear. The out-of-plane components of displacement gradient can be measured directly by utilizing collinear illumination and viewing from a direction normal to the object surface [8]. In practice collinear illumination and viewing is difficult experimentally so illumination at a small angle to the normal to the object surface is usually employed.

To measure the in-plane components of displacement gradient using shearography requires the measurement of the displacement gradient from a minimum of three directions of the sensitivity vector such that each of the measured components of displacement gradient contains a contribution from the out-of-plane and the in-plane components. The in-plane and out-of-plane components may then be calculated by a coordinate transformation. This coordinate transformation is subject to numerical errors unless the relative positions of the sensitivity vectors of the measured components are chosen carefully [8]. Also the variation in the sensitivity vector required precludes the measurement of in-plane displacement gradient using shearography from a single illumination and viewing direction.

The applied shear is determined by the direction and magnitude of the tilt applied to the shearing mirror in the shearing interferometer. Two orthogonal directions of applied shear are required to measure the displacement gradient in two directions, usually horizontally and vertically, across the object surface [6]. The magnitude of the applied shear controls the measurement sensitivity and is typically of the order of 2 to $10 \mathrm{~mm}$.

Phase-stepping techniques [3] can be used to extract the phase information, and therefore the displacement gradient, from the speckle interferograms.

\section{Theory - Optical Flow Field Technique}

Many image process techniques have been developed over the last decade or so to measure the surface displacement, and the general impression of these techniques is that they all come from the same mathematical process - correlation [4]. Besides the large amount of computational time that is involved, 
discrete correlation has the following mathematical issues that require further investigation:

- The size of the corresponding window is sensitive to the local deformation in the image. A smaller window size could increase the chance of mismatch; however, a larger window would prevent tiny local image deformations from being detected. The detection of small local displacement differences is crucial to the local strain estimation.

- The precision of the displacement estimation is important for the strain estimation since the local strain is the derivative of the local displacement. Working with digital images, interpolation either prior or post to the correlation process needs to be carried out to achieve a sub-pixel accuracy in the displacement gradient estimation.

The above issues are the inherent characteristics that could prevent all correlation based displacement estimation techniques from being effectively applied for strain estimation $[9,10,11]$.

The Optical Flow Field (OFF) is conventionally defined as the displacement distribution of the apparent motions of the brightness pattern on the image plane of an observer [5]. The OFF technique has developed significantly over the last twenty years.

All of the OFF estimation techniques are based on the assumption that the change in pixel intensity values is due to the motion of the speckle pattern, which is the projection of the environment. It follows that for corresponding points on the image plane:

$$
E(x, y, \quad t)=E(x+u, \quad y+v, \quad t+\Delta t)
$$

where $E(x, y, t)$ is the brightness of the point at $(x, y)$ on the image plane at time $t$, and the time interval separating the images, $\Delta t$, is the unit time period. Two statements are combined in equation (1): (a) the projections of the same object point located at $(x, y)$ on the image plane at time $t$ and moved to $(x+u$, $y+v$ ) at time $t+\Delta t$, and (b) that the brightness of these two image points should remain constant. Using the chain rule for differentiation, equation (1) may be expanded to give:

$$
E_{x} u+E_{y} v+E_{t}=0
$$

when $\Delta t=1$ and where $E_{x}=\partial E / \partial x, E_{y}=\partial E / \partial y$ and $E_{t}=\partial E / \partial t$. Equation (2) provides only a constraint on the brightness, the brightness constant constraint, on the two unknown parameters $u$ and $v$. The technique also requires a second constraint, which normally is a smoothness constraint. The smoothness constraint was proposed by Horn and Schunck [5], and related methods [12] are based on the assumption in which neighbor points on the viewed object have similar velocities and that the velocity field of the image brightness patterns varies smoothly almost everywhere. This is formally expressed by minimizing the square of the magnitude of the gradient of flow velocity. Obviously, only when a rigid object with smooth continuous surface is viewed could this assumption be valid. Fu and Pridmore [13] investigated the problem of a discontinuous surface by proposing a directional smoothness constraint to replace the traditional smoothness constraints. The directional smoothness constraint suggests that the flow field should be smooth over the image, but in terms of its' direction rather than of its' magnitude. This concept has been proved to be much more appropriate for fluid fields which can be considered as completely deformable objects [14]. In the situation where small local deformations need to be measured, we believe that the directional smoothness constraint is more suitable than any other form of numerical constraint to be applied for the analysis procedure. 
A measure of the departure from brightness constant smoothness in the image pair is given by:

$$
\xi_{b}=E_{x} u+E_{y} v+E_{t}
$$

Whilst a measure of the departure from directional smoothness in the image flow field is given by:

$$
\xi_{c}{ }^{2}=\left(\frac{\partial(u / v)}{\partial x}\right)^{2}+\left(\frac{\partial(u / v)}{\partial y}\right)^{2}
$$

The total error to be minimized is therefore:

$$
\xi^{2}=\iint \lambda^{2} \xi_{\mathrm{c}}^{2}+\xi_{\mathrm{b}}^{2} d x d y
$$

The task of estimating $u$ and $v$ becomes one of minimizing the error measure given in equation (5). The details of the procedure is given by Fu and Pridmore [13] and the iterative solution is summarized below:

$$
u_{n+1}=\bar{u}_{n}-\left(v_{n}^{2}+1\right) E_{x}\left(E_{x} \bar{u}_{n}+E_{y} v_{n}+E_{t}\right) /\left(E_{x}^{2} v_{n}^{2}+E_{y}^{2} u_{n}^{2}+\lambda^{2}\right)
$$

$$
v_{n+1}=\bar{v}_{n}-\left(u_{n}^{2}+1\right) E_{y}\left(E_{x} u_{n}+E_{y} \bar{v}_{n}+E_{t}\right) /\left(E_{x}^{2} v_{n}^{2}+E_{y}^{2} u_{n}^{2}+\lambda^{2}\right)
$$

where

$$
\begin{aligned}
\bar{u} & =\frac{1}{(4+2 \sqrt{2})}(u(x-1, y)+u(x+1, y)+u(x, y-1)+u(x, y+1)) \\
& +\frac{1}{(4+4 \sqrt{2})}(u(x-1, y-1)+u(x+1, y+1)+u(x+1, y-1)+u(x-1, y+1))
\end{aligned}
$$

and

$$
\begin{aligned}
\bar{v} & =\frac{1}{(4+2 \sqrt{2})}(v(x-1, y)+v(x+1, y)+v(x, y-1)+v(x, y+1)) \\
& +\frac{1}{(4+4 \sqrt{2})}(v(x-1, y-1)+v(x+1, y+1)+v(x+1, y-1)+v(x-1, y+1))
\end{aligned}
$$

The $\bar{u}$ and $\bar{v}$, are the local weighted average values of $u$ and $v$, at any arbitrary point $p(x, y)$. The separation distance between $p(x, y)$ and $p(x+1, y)$ is 1 pixel and between $p(x, y)$ and $\mathrm{p}(\mathrm{x}+1, \mathrm{y}+1)$ is $\sqrt{2}$ pixels.

$$
\begin{aligned}
& E_{x}=\frac{1}{4}(E(x+1, y, t)-E(x, y, t)+E(x+1, y+1, t)-E(x, y+1, t) \\
& \quad+E(x+1, y, t+1)-E(x, y, t+1)+E(x+1, y+1, t+1)-E(x, y+1, t+1))
\end{aligned}
$$




$$
\begin{aligned}
E_{y}= & \frac{1}{4}(E(x, y+1, t)-E(x, y, t)+E(x+1, y+1, t)-E(x+1, y, t) \\
& +E(x, y+1, t+1)-E(x, y, t+1)+E(x+1, y+1, t+1)-E(x+1, y, t+1)) \\
E_{t}= & \frac{1}{4}(E(x, y, t+1)-E(x, y, t)+E(x+1, y, t+1)-E(x+1, y, t) \\
& +E(x, y+1, t+1)-E(x, y+1, t)+E(x+1, y+1, t+1)-E(x+1, y+1, t))
\end{aligned}
$$

\section{Theory - Multi-Component Surface Strain Measurement}

The surface strain is determined from the six components of displacement gradient. Shearography is able to measure all the surface strain components [6]. Speckle pattern photography is able to measure the in-plane displacements from a single viewing direction [4] and these displacements may be differentiated numerically to yield the displacement gradient components $\delta \mathrm{u} / \delta \mathrm{x}, \delta \mathrm{v} / \delta \mathrm{x}, \delta \mathrm{u} / \delta \mathrm{y}$ and $\delta \mathrm{v} / \delta \mathrm{y}$. Speckle Pattern Photography is able to measure the in-plane and out-of-plane surface strain components using a stereoscopic technique utilizing two viewing directions [15]. This stereoscopic technique utilizes a coordinate transformation.

The strain tensor, $\mathrm{S}$, is given by:

$$
S=\left[\begin{array}{ccc}
\frac{\partial u}{\partial x} & \frac{1}{2}\left(\frac{\partial u}{\partial y}+\frac{\partial v}{\partial x}\right) & \frac{1}{2}\left(\frac{\partial u}{\partial z}+\frac{\partial w}{\partial x}\right) \\
\frac{1}{2}\left(\frac{\partial v}{\partial x}+\frac{\partial u}{\partial y}\right) & \frac{\partial v}{\partial y} & \frac{1}{2}\left(\frac{\partial v}{\partial z}+\frac{\partial w}{\partial y}\right) \\
\frac{1}{2}\left(\frac{\partial w}{\partial x}+\frac{\partial u}{\partial z}\right) & \frac{1}{2}\left(\frac{\partial w}{\partial y}+\frac{\partial v}{\partial z}\right) & \frac{\partial w}{\partial z}
\end{array}\right]
$$

where $u$ and $v$ are in-plane displacements in the $x$ and $y$ directions respectively, and $w$ is an out-of-plane displacement in the $z$ direction. The combined shearography and speckle pattern photography instrument utilises single-axis illumination and viewing. Shearography is used to determine the $\delta w / d x$ and $\delta w / d y$ components, with a typical measurement range of $\pm 500 \mu \varepsilon$. Speckle pattern photography is used to measure the in-plane components $\delta \mathrm{u} / \delta \mathrm{x}, \delta \mathrm{v} / \delta \mathrm{x}, \delta \mathrm{u} / \delta \mathrm{y}$ and $\delta \mathrm{v} / \delta \mathrm{y}$, with a typical measurement range of \pm $10000 \mu \varepsilon$. Neither shearography nor speckle pattern photography are able to measure the bulk strain components, $\delta \mathrm{u} / \delta \mathrm{z}, \delta \mathrm{v} / \delta \mathrm{z}$ and $\delta \mathrm{w} / \delta \mathrm{z}$ as both techniques can only determine properties at the object surface.

\section{Experimental}

The combined shearography and SPP instrument is composed of an out-of-plane sensitive shearography system, with a facility to perform measurements using two directions of applied shear, with additional software to perform the speckle pattern photography analysis. The experimental layout is shown in Fig. 1. 
Object

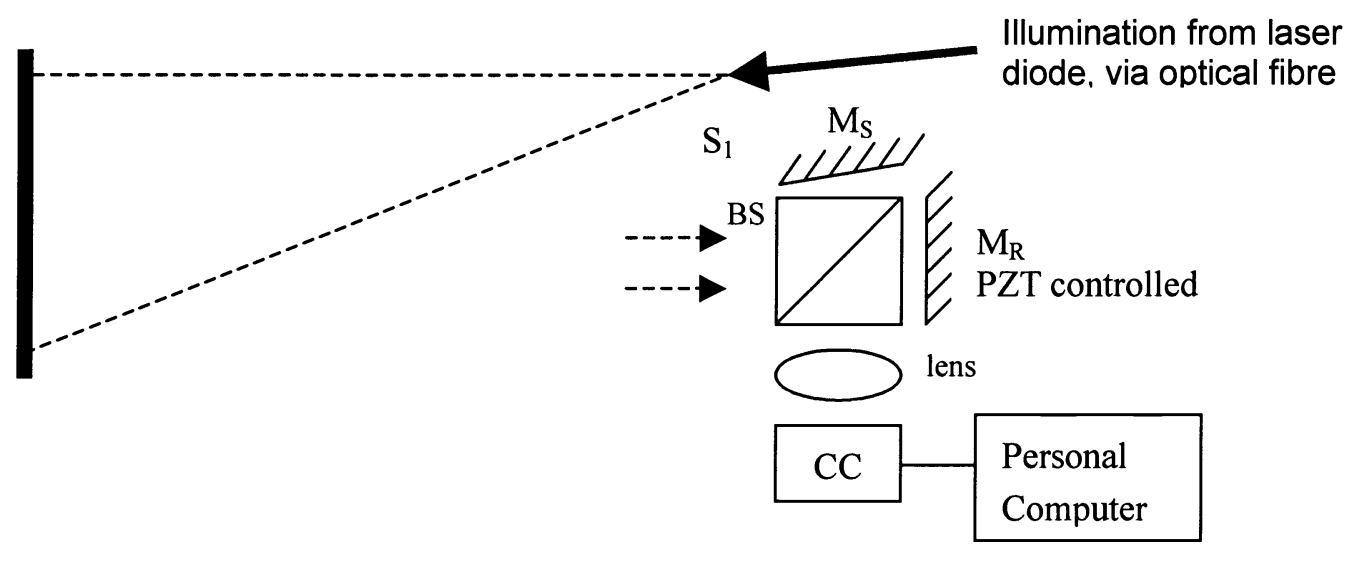

Fig. 1 Experimental layout of the combined speckle pattern photography and shearography instrument. In the instrument the illumination is arranged to be as close to the normal of the object surface, without obstructing the field of view of the camera. BS, beam-splitter; $M_{S}$, shearing mirror, $M_{R}$, reference mirror

A fibre Bragg grating stabilized laser diode $(810 \mathrm{~nm}, 100 \mathrm{~mW})$ with fibre beam delivery to the source position was used to illuminate the object. The laser diode output wavelength is stabilized using the light reflected by the fibre Bragg grating feeding back into the laser cavity. The fibre beam delivery provides spatial filtering of the beam and a lens at the distal end of the fibre allows adjustment of the expansion of the beam illuminating the object under investigation. The beam expansion lens was located $600 \mathrm{~mm}$ from the object and $90 \mathrm{~mm}$ below the normal to the center of the object surface.

The interferometer head is composed of a shearing Michelson interferometer, a camera lens and a CCD camera. The shearing interferometer is composed of a beam-splitter, a shearing mirror that could be adjusted to give varying magnitudes and directions of applied shear and a reference mirror that was fitted with a piezoelectric transducer (PZT) to allow phase-stepping to be performed. A PC controls the image capture and phase stepping using a program written in $\mathrm{LabVIEW}^{\mathrm{TM}}$.

To perform a measurement of the full surface strain of an object undergoing deformation requires the collection of images before and after object deformation. Initially the shear is applied in the horizontal direction. The object is illuminated using the laser with the interferometer shearing mirror which blocked to prevent the formation of interferometric speckles, a reference SPP image is recorded. The shearing mirror is unblocked and a reference interferometric speckling pattern recorded by the camera. The object is deformed and three phase-stepped interferometric speckle patterns are recorded. These are combined using the LabVIEW ${ }^{\mathrm{TM}}$ program to obtain a wrapped phase map of the displacement gradient for that direction of applied shear. The shearing mirror is again blocked and a second SPP image is recorded. The speckle pattern photography images are processed using software written within the Center for Computer Vision and Image Processing. The phase-stepped images recorded by the shearography technique are processing using ISTRA phase unwrapping software [16].

\section{Results}

A flat plate test object, which clamped around the perimeter and painted with white paint (Dulux vinyl matt), was deformed using a micrometer screw gauge which located approximately in the center of the field. The deformation had a magnitude of a few micrometers and was normal to the object surface. The 
speckle patterns recorded before and after deformation, which were used for the SPP analysis, are shown in Fig. 2. The wrapped phase maps recorded from the shearography instrument are shown in Fig. 3.

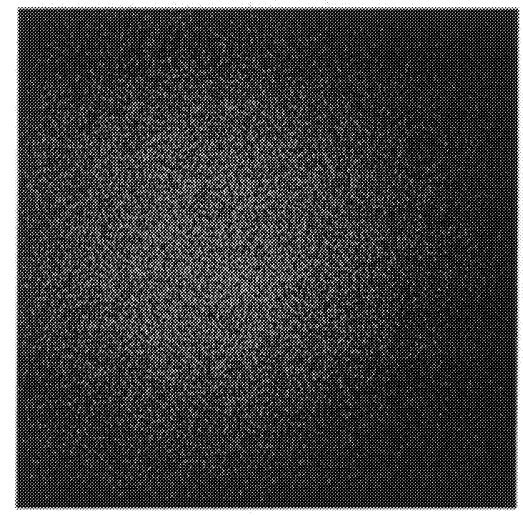

(a)

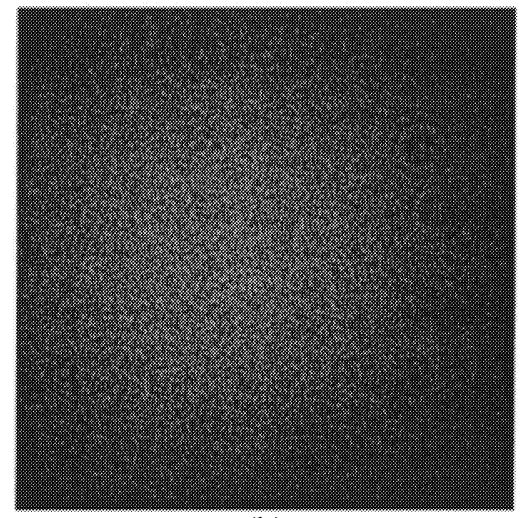

(b)

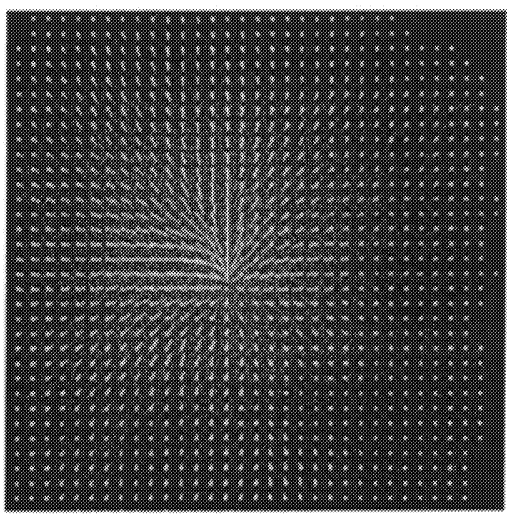

(c)

Fig. 2 Non-interferometric speckle patterns recorded (a) before and (b) after application of a point displacement, of a few micrometers, normal to the surface of a flat plate test object. These speckle patterns were used to obtain the displacement field, shown in (c), using the SPP analysis technique.

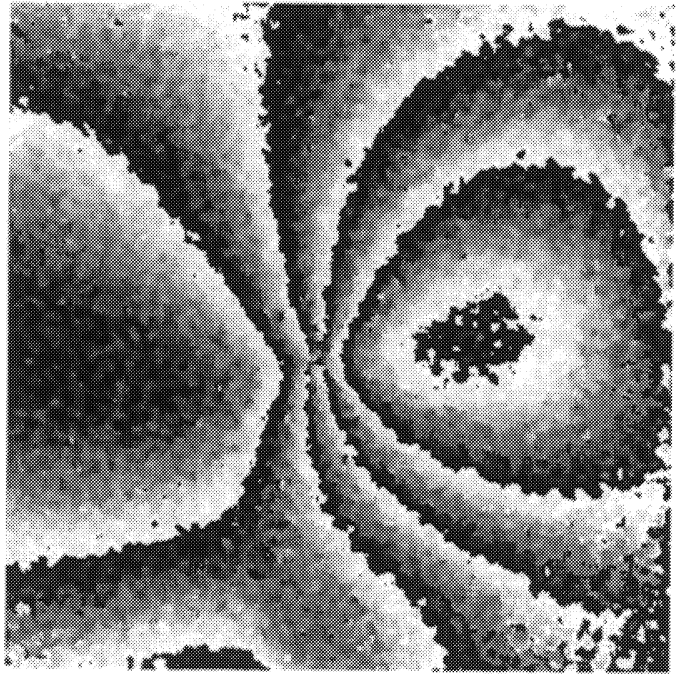

(a)

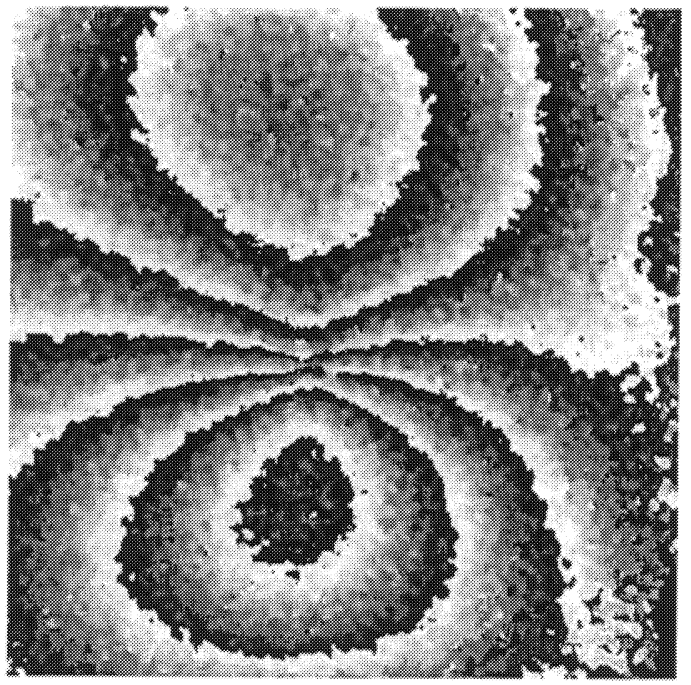

(b)

Fig. 3 Wrapped phase maps recorded from the shearography system of a point displacement, of a few micrometers, normal to the surface of a flat plate test object. They are (a) with a horizontal applied shear of $2.5 \mathrm{~mm}$ and (b) with a vertical applied shear of $2.5 \mathrm{~mm}$. 


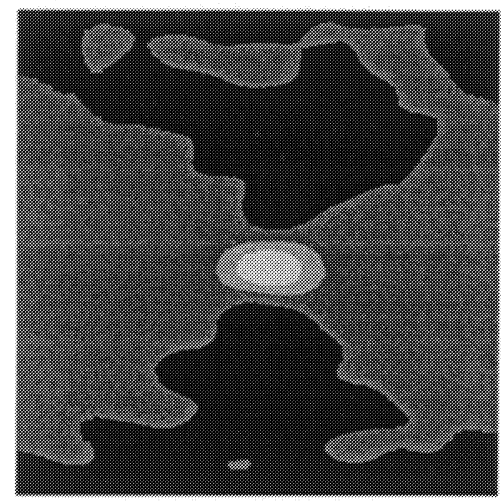

(a)

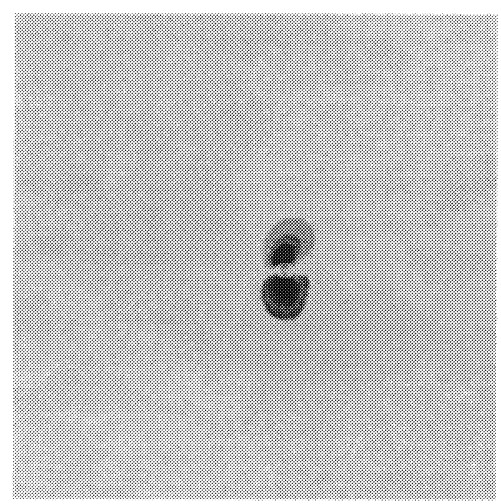

(d)

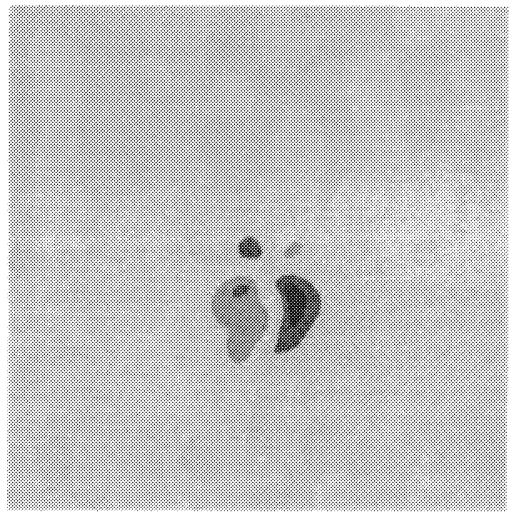

(b)

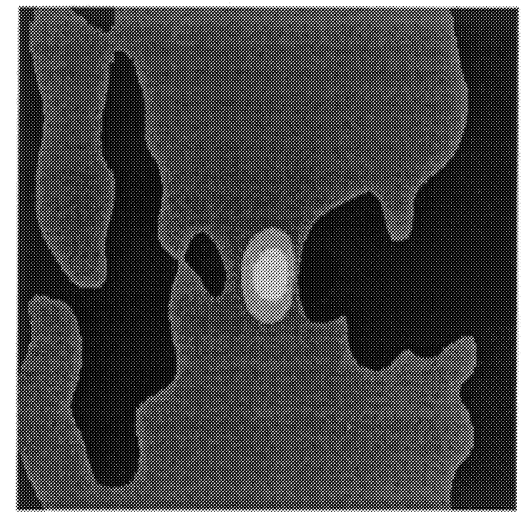

(e)

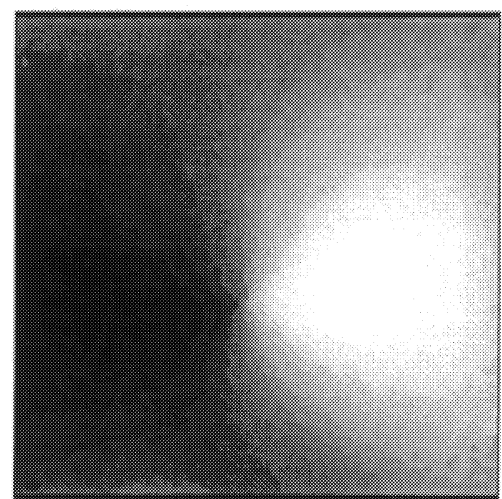

(c)

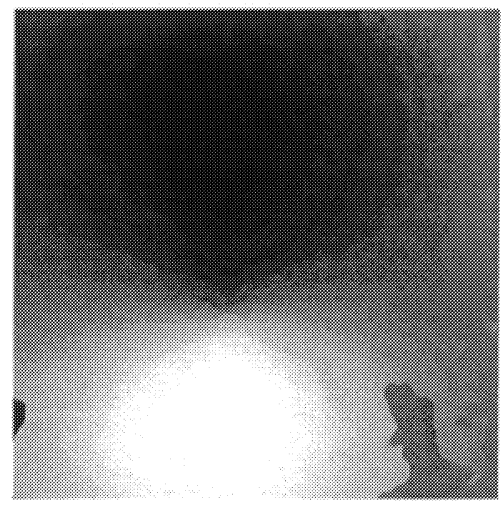

(f)

Fig. 4 The six components of displacement gradient measured using the combined shearography/SPP instrument. The field of view is approximately $150 \mathrm{~mm}$ by $150 \mathrm{~mm}$. (a) and (b) show displacement gradients between $\quad-4000 \mu \varepsilon$ (black) and $+8000 \mu \varepsilon$ (white) for the $\delta u / \delta x$ and the $\delta v / \delta x$ components respectively. (d) and (e) show displacement gradients between $-1000 \mu \varepsilon$ (black) and $+8000 \mu \varepsilon$ (white) for the $\delta u / \delta y$ and the $\delta v / \delta y$ components respectively. (c) and (f) show displacement gradients between $-500 \mu \varepsilon$

(black) and $+\mathbf{5 0 0}$ $\mu \varepsilon$ (white) for the $\delta w / \delta x$ and the $\delta w / \delta y$ components respectively.

The speckle patterns, shown in Fig 2, are processed using the optical flow field technique, described in Section 3 , to yield the in-plane displacement gradient components, $\delta u / \delta x, \delta v / \delta x, \delta u / \delta y$ and $\delta v / \delta y$. Unwrapping of the wrapped phase maps shown in Fig 3 yields the out-of-plane displacement gradient components $\delta \mathrm{w} / \delta \mathrm{x}$ and $\delta \mathrm{w} / \delta \mathrm{y}$. The six components of displacement gradient that are required to fully characterise the surface strain, are shown in Fig 4.

The results presented in Fig. 4 are of an area of $150 \mathrm{~mm}$ by $150 \mathrm{~mm}$ on the surface of the flat plate. The measurement range is from $-4000 \mu \varepsilon$ to $+8000 \mu \varepsilon$ for the $\delta u / \delta x$ and the $\delta v / \delta x$ components. The measurement range is from $-1000 \mu \varepsilon$ to $+8000 \mu \varepsilon$ for the $\delta u / \delta y$ and the $\delta v / \delta y$ components. The measurement range is from $-500 \mu \varepsilon$ to $+500 \mu \varepsilon$ for the $\delta w / \delta x$ and $\delta w / \delta y$ components. 


\section{Discussion}

The combined SPP and shearography technique provides a method to fully characterize the surface strain using illumination and viewing from a single direction, using the same hardware that is required for single channel shearography. The single axis illumination and viewing allows full characterization of surface strain of engineering objects where optical access is insufficient for multi-component shearography [8] to be performed.

This combined SPP and shearography technique also provides independent direct measurements of the individual displacement gradient components. One of the disadvantages of the measurement of displacement gradient using shearography is that in-plane components are determined by combining measurements from two, or more channels. Similarly for SPP the out-of-plane displacement gradient component can determine using a stereoscopic technique, again combining measurements from two channels. These multi-channel measurements are prone to errors due to the coordinate transformation and contain error contributions from the two measured channels. The combined SPP and shearography technique presented in this paper overcomes these difficulties.

\section{Conclusions}

In this paper a single-access multi-component surface strain measurement instrument has been demonstrated. The instrument combines the speckle interferometry technique of shearography and the image processing technique of speckle pattern photography to fully characterize the surface strain.

\section{Acknowledgments}

The authors acknowledge support from the Engineering and Physical Sciences Research Council, UK, Agusta Westland and the Civil Aviation Authority, UK, under project GR/N11872/01.

\section{References}

[1] Leendertz J. A., Butters J. N., "An image-shearing speckle pattern interferometer for measuring bending moments ", J. Phy. E., Vol. 6, (1973), pp. 1107-1110.

[2] Hung Y. Y., "Shearography: a new optical method for strain measurement and nondestructive testing", Opt. Eng., Vol. 21, No. 3, (1982), pp. 391-395.

[3] Creath K., "Temporal phase measurement methods" in "Interferogram analysis, digital fringe measurement techniques", ed. Robinson D. W. and Reid G. T., Institute of Physics, Bristol, (1993).

[4] Sjödahl M. and Benckert L. R., "Electronic speckle photography: analysis of an algorithm giving the displacement with subpixel accuracy", Appl. Opt., Vol. 32, (1994), pp. 2278-2284.

[5] Horn B. K. P. and Schunck B. G., "Determining optical flow", Journal of Artificial Intelligence, Vol. 17, (1981), pp.185-203.

[6] Aebischer H. A. and Waldner S., "Strain distributions made visible with image-shearing speckle pattern interferometry”, Opt. Laser. Eng., Vol. 26, (1997), pp. 407-420.

[7] Hung Y. Y. and Liang C. Y., "Image-shearing camera for direct measurement of surface strains", Appl. Opt., Vol. 18, No. 7, (1979), pp. 1046-1051. 
[8] James S. W. and Tatam R. P., "Time-division-multiplexed 3D shearography", Proc. SPIE 3744, (1999), pp. 394-403.

[9] Vendroux G. and Knauss W. G., "Submicron deformation field measurement: Part 2. Improved digital image correlation”, Expt. Mech., Vol. 38, No. 2, (1998), pp. 86-92.

[10]Manthey D. W. and Lee D., "Vision-based surface strain measurement system", JOM., Vol. 47, no. 7, (1995), pp. 46-49.

[11]Chu T. C., Ranson W. F., Sutton M. A. and Peters W. H., "Applications of digital-image-correlation techniques to experimental mechanics ”, Expt. Mech., September (1985), pp. 232-244.

[12]Uras S., Girosi F., Verri A. and Torre V., "A computational approach to motion perception”, Biological Cybernetics., Vol. 60, (1988), pp79-87.

[13]Fu S. and Pridmore T. P., "Image flow field detection", Third International Conference on Signal Processing (ICSP'96), Beijing, Vol. 2, (1996), pp.1090-1093.

[14]Fu S. and Wu Y., "Quantitative analysis of velocity distribution from Schlieren images", The Proceeding of 8th International Symposium on Flow visualization, Sorrento, Italy, Sept. 1-4, (1998).

[15]Larsson L., "Stereo speckle microscopy for 3D-deformation fields," Symposium on Optical Metrology Techniques for Industrial Applications, Grasmere, UK, (2002).

[16]Ettemeyer A., Neupert U., Rottenkolber H. and Winter C., "Schnelle und robuste bildanalyse von streifenmustern-ein wichtiger schritt der automation von holografishen prozessen", $1^{\text {st }}$ International Workshop on Automatic Processing of Fringe Patterns, (1989), pp. 23-31.

R. M. Groves ${ }^{1}$, S. Fu ${ }^{2}$, S. W. James ${ }^{3}$, R. P. Tatam ${ }^{4}$

${ }^{1,3,4}$ Optical Sensors Group, Centre for Photonics and Optical Engineering, ${ }^{2}$ Centre for Computer Vision and Image Processing School of Engineering, Cranfield University, Cranfield, Bedfordshire, MK43 0AL, UK

E-mail address: s.w.james@cranfield.ac.uk 